\title{
Role of adenosine deaminase 2 gene variants in pediatric deficiency of adenosine deaminase 2: A structural biological approach
}

\author{
MARIA I. ZERVOU ${ }^{1}$, GEORGE N. GOULIELMOS ${ }^{1}$, MICHAIL MATALLIOTAKIS ${ }^{1-3}$, \\ CHAROULA MATALLIOTAKI ${ }^{1-3}$, DEMETRIOS A. SPANDIDOS ${ }^{4}$ and ELIAS ELIOPOULOS ${ }^{5}$
}

\begin{abstract}
${ }^{1}$ Section of Molecular Pathology and Human Genetics, Department of Internal Medicine, School of Medicine, University of Crete, 71003 Heraklion; ${ }^{2}$ Third Department of Obstetrics and Gynecology, Aristotle University of Thessaloniki, 54124 Thessaloniki; ${ }^{3}$ Department of Obstetrics and Gynecology, Venizeleio and Pananio General Hospital of Heraklion, 71409 Heraklion; ${ }^{4}$ Laboratory of Clinical Virology, School of Medicine, University of Crete, 71003 Heraklion;

${ }^{5}$ Laboratory of Genetics, Department of Biotechnology, Agricultural University of Athens, 11855 Athens, Greece
\end{abstract}

Received October 23, 2019; Accepted December 4, 2019

DOI: $10.3892 / \mathrm{mmr} .2019 .10862$

\begin{abstract}
Adenosine deaminase 2 (ADA2) belongs to the novel family of adenosine deaminase growth factors (ADGFs), which play an important role in tissue development. The deficiency of adenosine deaminase 2 (DADA2) is a recently recognized autosomal recessive autoinflammatory disease, characterized by various systemic vascular and inflammatory manifestations, which is associated with ADA2 mutations. Considering that a recent screening of an international registry of children with systemic primary vasculitis revealed novel and already known variants in ADA2, this study aimed to further investigate the functional significance of the rare variants detected, namely p.Gly47Arg, p.Gly47Ala, p.Arg8Trp, p.Leu351Gln and p.Ala357Thr, by using a structural biological approach. Three-dimensional models of the mutants were developed and their three-dimensional (3D) structures were subjected to detailed interaction and conformational analyses. This led to suggestions that the novel mutations found may affect the formation/stability of the homodimer or may influence the activity of the enzyme. It was thus concluded that the Arg8Trp and Gly47Arg mutations affect the position and interaction of the dimer-associated HN1 helical structure and therefore, dimer formation and stabilization, while Leu351Gln and Ala357Thr influence the metal coordination in the active site. These findings shed further light onto the structural consequences of the mutations under investigation.
\end{abstract}

Correspondence to: Dr Maria I. Zervou, Section of Molecular Pathology and Human Genetics, Department of Internal Medicine, School of Medicine, University of Crete, 71003 Heraklion, Greece E-mail: mzervou@med.uoc.gr

Key words: adenosine deaminase 2, deficiency of adenosine deaminase 2 , gene polymorphism, three-dimensional model

\section{Introduction}

Deficiency of adenosine deaminase 2 (DADA2) is an autosomal recessive autoinflammatory disease, characterized by early-onset vasculopathy appearing a wide range of clinical manifestations, associated with mutations in adenosine deaminase 2 (ADA2) enzyme $(1,2)$. This condition is mainly characterized by an inflammatory vasculopathy resembling systemic polyarteritis nodosa (PAN) (3). DADA2 has been suggested to compromise endothelial integrity (2) or account for the development of Sneddon's syndrome in some patients, which represents a poorly understood disorder most common among middle-age women (4). Patients with DADA2 are characterized by immunodeficiency of variable severity, which is most evident in B cells 2 (2). Hematological manifestations refer to pure red cell aplasia (PRCA), thrombocytopenia, neutropenia and a severe type of anemia (2,5-7). Other common manifestations include intermittent fever, arthralgia, lymphadenopathy and early-onset stroke. In the majority of cases, patients present with neurological manifestations in both the peripheral and central nervous system (CNS) (8). CNS involvement is very frequent, as presented by brain MRI studies that have revealed chronic ischemic lesions in the basal ganglia, thalami and pons $(3,9-11)$.

ADA2, a 59-kDa enzyme, consisting of 511 amino acid residues, is encoded by the $A D A 2$ locus, formerly known as the cat eye syndrome chromosome region, candidate 1 (CECRI) gene located on chromosome 22 (1). It forms homodimers and is secreted (by antigen-presenting cells) with high expression levels in plasma. ADA2 is considered to be critical for the maintenance of vascular integrity, and to be responsible for the extracellular degradation of adenosine. It has also been implicated in the regulation of proliferation of activated $\mathrm{T}$ cells and macrophages, as well as in the differentiation of monocytes to macrophages $(2,12)$. Moreover, it has been found that ADA2 can bind to different cell types through proteoglycans and with a higher specificity to $\mathrm{T}$ cells via an unknown receptor (13). Adenosine is an important signaling molecule and is normally 
found in low concentrations which may increase significantly as a result of cell damage, inflammation, oncogenesis and hypoxia (14). ADA2 plays a role in a high spectrum of disorders, connecting systemic inflammation, vascular pathology, and mild immunodeficiency (2). It is a highly polymorphic gene and >300 missense substitutions and insertions/deletions (indels) have been identified thus far. Importantly, a high number of copy number variants (CNVs) have been detected across the ADA2 coding gene (http://exac.broadinstitute. org; http://gnomad.broadinstitute.org). However, as regards DADA2, 61 disease-causing mutations have been described to date, located over the entire coding region of ADA2, with the majority of these being missense variants, although genomic deletions and nonsense mutations have also been reported (15).

ADA2 belongs to the novel family of adenosine deaminase growth factors (ADGFs), which play an important role in tissue development. The three-dimensional (3D) structures of ADA2 reveal the structural basis of the catalytic/signaling activity of ADGF/ADA2 proteins. The structure is composed of an 8 -stranded, parallel $\beta$-sheet that closes into a barrel and is surrounded by classical $\alpha / \beta$-TIM barrel motif helices and six additional $\alpha$-helices located at the $\mathrm{N}$-terminus ( $\mathrm{NH} 1, \mathrm{NH} 2$, $\mathrm{NH} 3$ and NH4) and at the $\mathrm{C}$ terminus (H4 and H5) (Fig. 1). Loops between $\beta$-strands and $\alpha$-helices constitute most of the essential features of the catalytic site. ADA2 consists of 4 domains: The signal sequence, the dimerization domain, the putative receptor-binding (PRB) domain and the catalytic domain (3).

The ADA catalytic domain contains a deep oblong-shaped active site cavity lined by the $\mathrm{C}$-terminal segments and connecting loops of the $\beta$-barrel strands, acting as a 'floor' and 'walls', and are capped from above with a 'ceiling' composed of helices $\mathrm{H} 3$ and a7 and the hairpin loop between a7-1 and a7-2 helices (Figs. 1 and 2). The zinc ion sits in the deepest part of the active site cavity in the center of the C-terminal end of the barrel. It is coordinated to 4 invariant residues: His- 86 and His-88 in the b1 strand, His-330 in the b5 strand, and Asp-415 in the loop between b1 strand and a8-1 helix (Fig. 3, left panel). The discovery of the zinc ion confirms the earlier suggestion that ADGF/ADA2 as well as ADA1 family proteins are zinc-dependent hydrolases $(16,17)$.

In addition to the catalytic domain, the $3 \mathrm{D}$ structure has revealed two ADGF/ADA2-specific domains of novel folds that mediate the protein dimerization and binding to the cell surface receptors. The $\mathrm{N}$-terminal extension forms by the N-terminal $\alpha$-helices HN1, HN2, HN3 and HN4, whereas the C-terminal extension elongates the C-terminal helix H5, which together with the N-terminal helices forms a unique helix-turn-helix arrangement (Fig. 1). Two highly conserved charged residues of helix HN1, Arg-8 and Glu-15, are engaged in ionic interactions with the Asp-347 and His-365 of the neighboring subunit, respectively. An extensive glycosylation and the presence of a conserved disulfide bond and a signal peptide in ADA2 strongly suggest that ADA2, in contrast to ADA1, is specifically designed to act in the extracellular environment (12).

Recently, Gibson et al (18) performed the screening of an international registry of children with systemic primary vasculitis for variants in ADA2 and the subsequent genotyping of 9 children identified with DADA2. By performing
DNA sequencing of the coding exons, they found rare variants of either known (p.Gly47Arg and p.Gly47Ala) or novel (p.Arg8Trp, p.Leu351Gln and p.Ala357Thr) associations with DADA2. Moreover, they assessed the functional consequences of the identified variants by using specific ADA2 assays and immunoblotting. Prompted by these recent results, and considering the suggestion that screening ADA2 among children with vasculitis rash, unclassifiable vasculitis (UCV), PAN, or unexplained early-onset CNS disease with systemic inflammation may enable an earlier diagnosis of DADA2 (18), this study was performed in an attempt to further elucidate the functional significance of these mutations by using a structural biological approach.

\section{Materials and methods}

The three dimensional structure of human ADA2 in complex with coformycin, a transition state analog, (PDB code 3LGG) was downloaded from the Protein Data Bank and used to analyze the consequences to structure and function of the mutations p.Gly47Arg, p.Gly47Ala, p.Arg8Trp, p.Leu351Gln and p.Ala357Thr. Mutants were constructed using molecular modeling with the program Maestro (Schrodinger, LLC) which was also used to analyze the conformational changes caused by the mutation. Rotational flexibility on mutated side chains was tested due to the restricted space in the mutation vicinity and the conformation with the least bad contacts was adopted. The electrostatic surface potential of the models was calculated by the Adaptive Poisson-Boltzmann Solver (APBS) using the PyMOL plug-in with the default parameter settings. All figures depicting 3D models were created using the molecular graphics program PyMOL V.2.2 (19).

\section{Results}

PAN-associated mutations in ADA2 structure. Taking into account the domain description detailed in the 'Introduction' and the secondary structure elements involved in their functionality, the 5 PAN-associated mutants examined seem to be readily involved in functional changes.

Helix HN1 projects as a finger from its own subunit and almost entirely interacts with the ADA domain of the neighboring subunit (Fig. 4, left panel). This 'helix anchor' provides the major contact between subunits in the dimer that contributes $>40 \%$ of the hydrophobic interactive area. Helix HN1 docks to the surface created by helices a5 and a6. Two highly conserved charged residues of helix HN1, Arg-34 and Glu-41, are engaged in ionic interactions with the Asp-373 and Arg369 of the neighboring subunit, respectively (Fig. 4, left panel). Hydrophobic Ile-30, Leu-37, Leu- 38, as well as parts of aliphatic chains of polar Thr-33 and Lys-14 form hydrophobic contacts with residues of the neighboring subunit. A close examination of the interactions of the ADA2 dimer interface (Fig. 4A), is illustrating the residue contacts between the two HN1 helix anchors, where Arg34 (blue-gray) is located. The Arg34Trp PAN mutation (Fig. 4, right panel) causes severe clashes between the bulky side chain of the tryptophane 34 side chain and Leu372 of the homodimer's a5 helix as well as loss of the homodimer stabilizing hydrogen bond interaction (in yellow dashed lines) between Arg 34 (blue-gray) and 


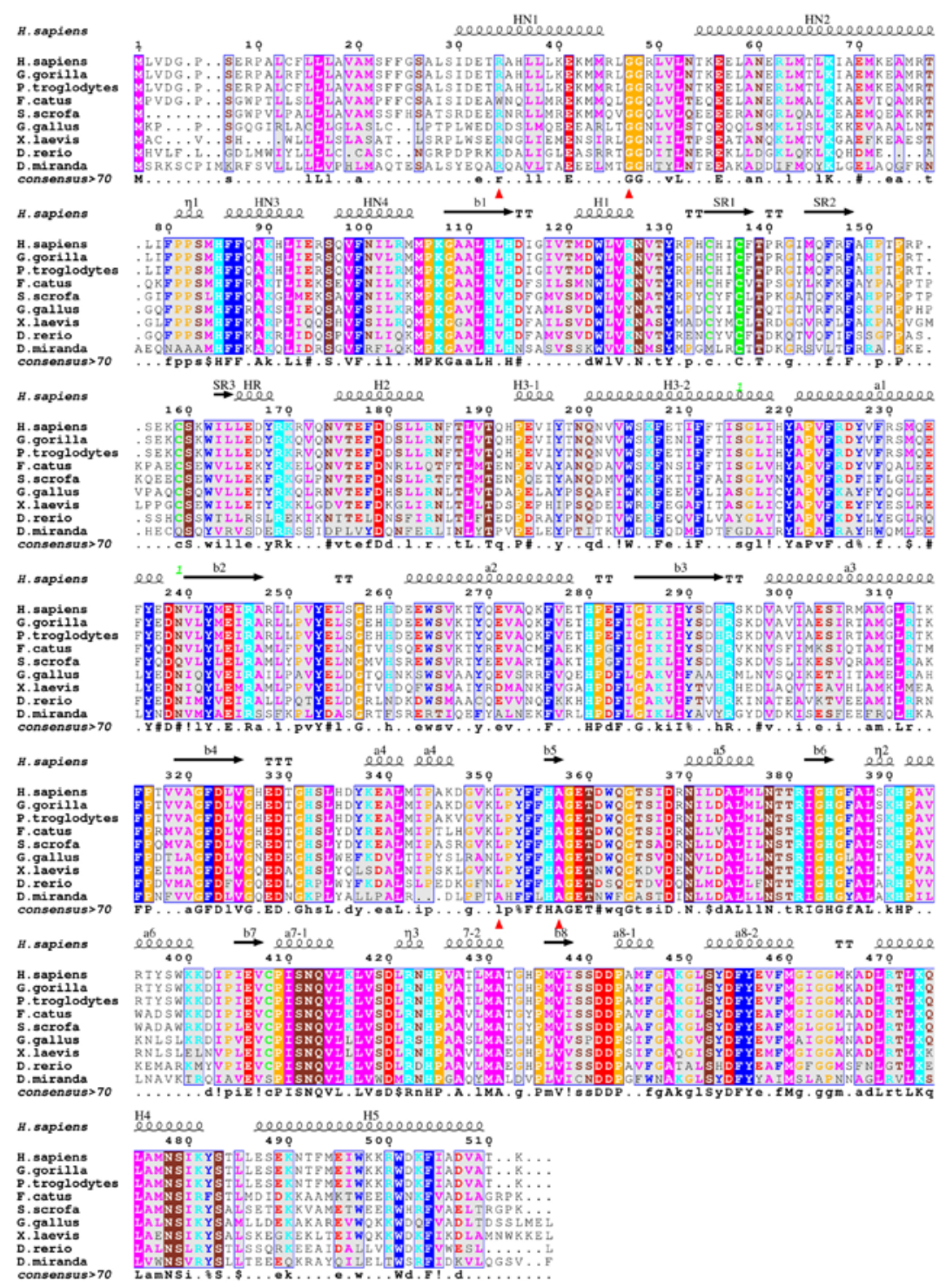

Figure 1. Multiple alignment of ADA2 protein sequences from diverge species and indication of the mutations in patients with PAN. Evolutionary conservation is indicated by capital letters in the consensus sequence and from the colored columns. Mutation positions are indicated by red triangles. Colored columns indicate amino acid physicochemical properties. Numbering is according to the complete human delaminate 2 sequence. ADA2, adenosine deaminase 2; PAN, polyarteritis nodosa.
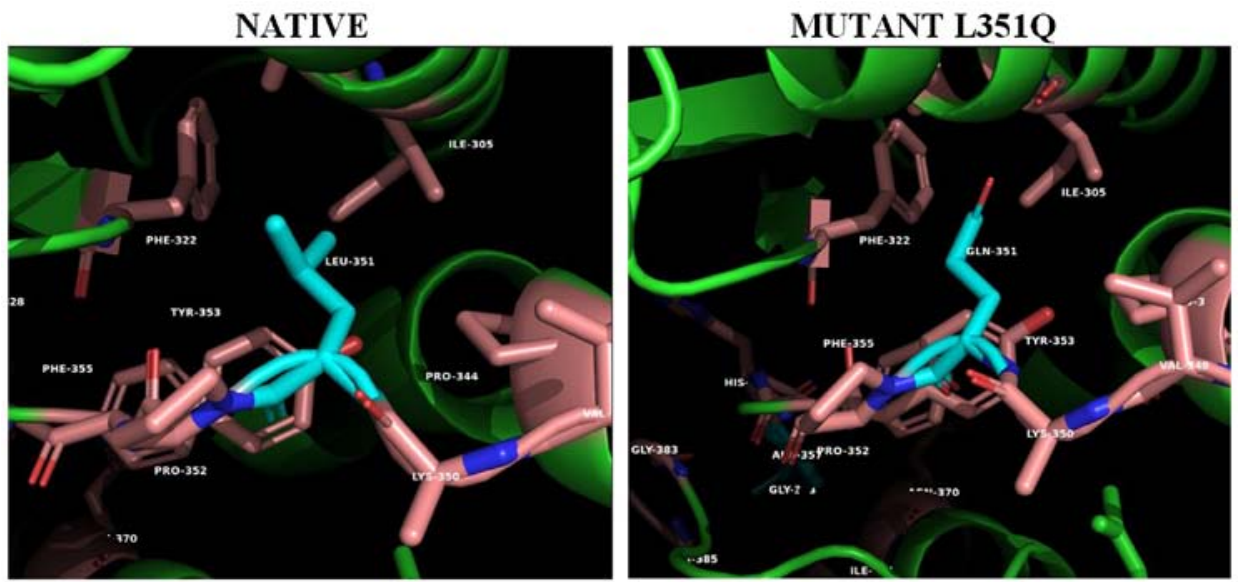

Figure 2. The PAN-associated novel mutation L351Q in the ADA2 structure. Panels illustrate the 3D native structure of ADA2 (PDB 3LGG) and mutant on position \#351. The hydrophobic intercalation between helices $\alpha 4$ (residues 306-321) and $\alpha 5$ (residues 341-351) is being interrupted with the introduction of the polar Gln351 in the Leu (orange, cyan)/Phe (cyan) formed hydrophobic pocket. This shift could be transmitted to the neighboring His 358 coordinated to the metal ion or affect the confirmed glycosylation at the next Asn352 (2). Structures were analyzed, mutants constructed and cartoons rendered using the PyMOL Molecular Graphics System, Version 2.2. ADA2, adenosine deaminase 2; PAN, polyarteritis nodosa. 

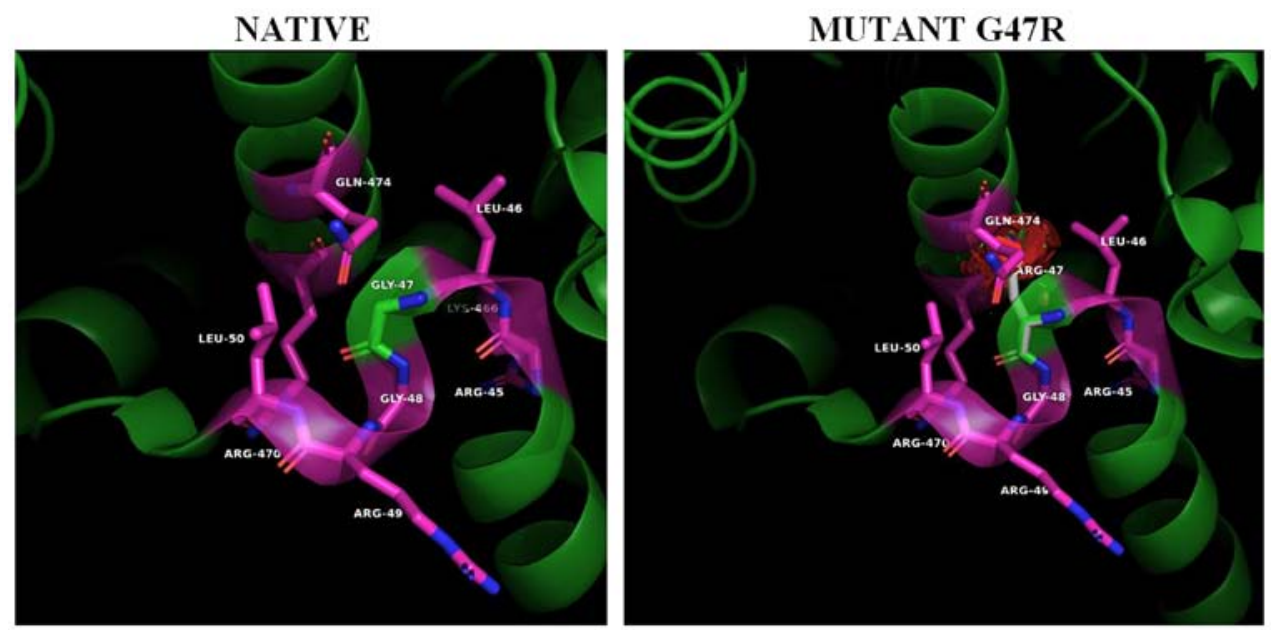

Figure 3. The PAN-associated novel mutation G47R in ADA2 structure. Panels show the 3D native structure of ADA2 (PDB 3LGG) and mutants around position \#47. Gly47 (green) is located in the $\mathrm{i}+2$ position of a tight double beta turn regulating the position of the N-terminal HN1 dimer association helix. Substitution of the small non-polar glycine with the much larger and positively charged arginine (white) will strongly alter the relative position of HN1 helix with respect to the $\alpha 5$ and $\alpha 6$ homo-monomer's interacting helices, reducing the stability of the subunits, weakening the monomer to monomer anchoring interactions and the formation of stable dimers. Structures were analyzed, mutants constructed and cartoons rendered using the PyMOL Molecular Graphics System, Version 2.2. ADA2, adenosine deaminase 2; PAN, polyarteritis nodosa.
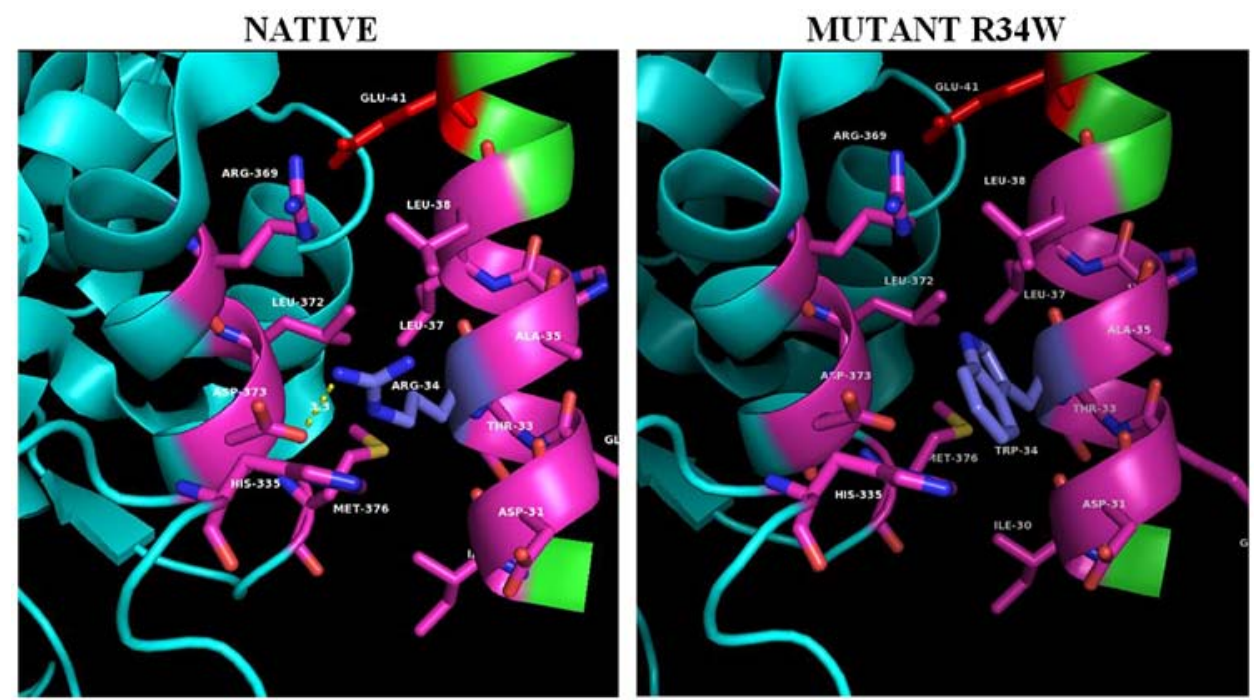

Figure 4. The PAN-associated novel mutation R34W in the ADA2 structure. Panels illustrate the 3D native structure of ADA2 (PDB 3LGG) and mutant on position \#8. A section of the ADA2 dimer interface is shown, illustrating the residue contacts between the two HN1 helix anchors, where Arg34 (blue-grey) is located. The Arg34Trp mutant causes severe clashes between the bulky side chain of the tryptophane 34 side chain and Leu 372 of the homodimer's a5 helix as well as loss of the dimer stabilizing hydrogen bond interaction (in yellow dashed lines) between Arg34 (blue-grey) and homomonomer Asp373 (red). Structures were analyzed, mutants constructed and cartoons rendered using the PyMOL Molecular Graphics System, Version 2.2. ADA2, adenosine deaminase 2; PAN, polyarteritis nodosa.

homomonomer Asp373 (purple). It is worth noting that in cat's native sequence where position 34 is occupied by a tryptophane residue the opposing homodimer's a5 helix residue 373 has been replaced by a leucine enhancing the hydrophobic interaction between HN1 and a5-helix.

Helix HN1 is followed by a sharp twist and 5-residue loop, positioned at the right angle relative to the helix. The twist and loop, as well as helices HN2, HN3 and HN4 pack on the surface of the ADA domain of own subunit. The loop residues have been observed with high conformational stability. Gly47 (Fig. 3, left panel) is located at the top of the tight turn between the HN1 and HN2 $\alpha$-helices that support dimerization. Specifically, the Gly47 amino acid residue (Fig. 3, left panel) is located in the $\mathrm{i}+2$ position of a tight double $\beta$ turn regulating the position and angle of the N-terminal HN1 dimer association helix (12). The substitution of the small non-polar glycine with the much larger and positively charged arginine (Fig. 3, right panel) will strongly alter the relative position of $\mathrm{HN} 1$ helix with respect to the a5 and a6 homo-monomer's interacting helices, reducing the stability of the subunits, weakening the monomer to monomer anchoring interactions and the formation of stable dimers (20).

The hydrophobic cluster between helix a4 (residues 338-348) and strand b5 (residues 349-356) is being interrupted with the introduction of the polar Gln351 (PAN 

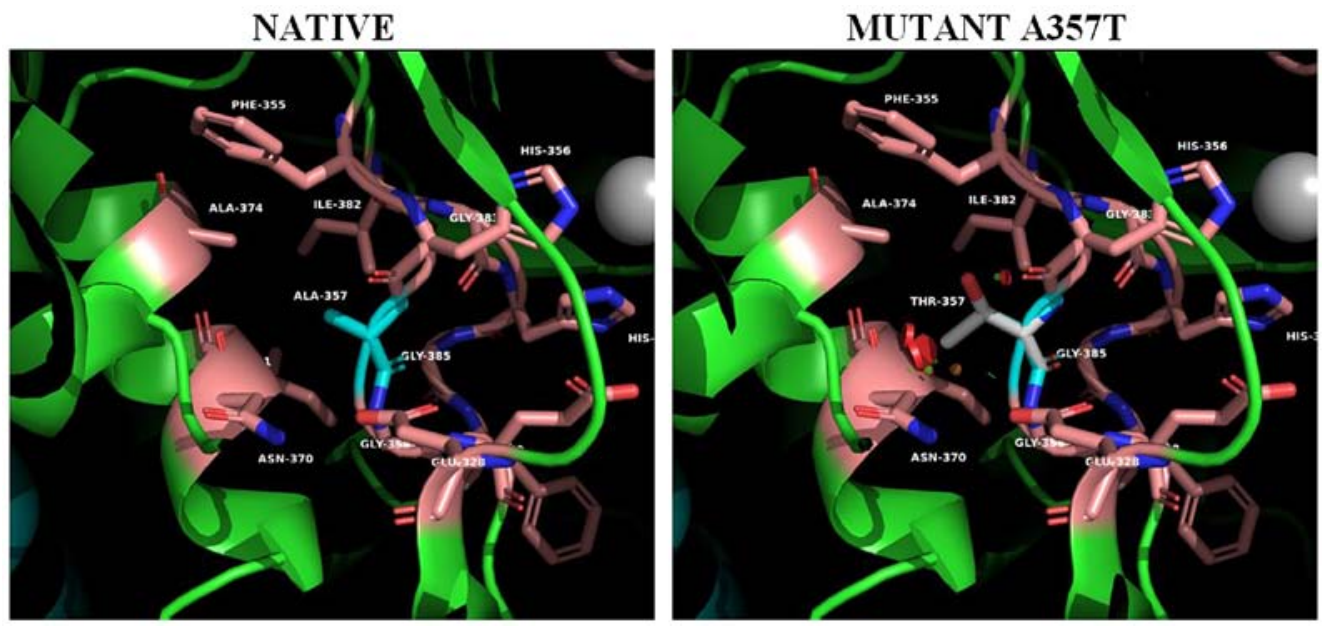

Figure 5. The PAN-associated novel mutation A357T the in ADA2 structure. Panels illustrate the 3D native structure of ADA2 (PDB 3LGG) and mutant on position \#357. The spatial distortion introduced to the metal coordination site is even more apparent. The introduction of the polar threonine side chain located on a beta-bulge (res.357-358) and in contact with the metal coordinated His 356 further distorts metal coordination at the active site influencing the activity of the enzyme. Structures were analyzed, mutants constructed and cartoons rendered using the PyMOL Molecular Graphics System, Version 2.2. ADA2, adenosine deaminase 2; PAN, polyarteritis nodosa.

mutant L351Q) in the Ile305/Phe322-formed hydrophobic pocket (Fig. 2). This disruption could be transmitted to the neighboring His356 coordinated to the metal ion or affect the confirmed glycosylation at the next amino acid residue Asn352 (12).

For the mutant on position 357 (Ala357Thr) (Fig. 5), the spatial distortion introduced to the metal coordination site is even more apparent. The introduction of the polar threonine side chain located on a $\beta$-bulge (res.356-357) and in contact with the metal coordinated His 356 further distorts metal coordination at the active site influencing the activity of the enzyme. In addition this mutation creates unfavorable bad contacts with helix $\alpha 5$.

\section{Discussion}

In the present study, the authors sought to analyze the functional role of rare variants of either known (p.Gly47Arg and p.Gly47Ala) or novel (p.Arg34Trp, p.Leu351Gln and p.Ala357Thr) of the ADA2 protein, by using 3-D modeling and assessing the structural consequences of the respective amino acid substitutions. The identified variants showing a novel association with DADA2 were initially predicted to be damaging (18). ADA2 has been considered to be both the major extracellular adenosine deaminase and an adenosine deaminase-related growth factor (1). Analysis of the 3D protein structures of ADA2 conducted by our group, regarding either already known or novel mutations suggested that the novel mutations found may affect the formation/stability of the homodimer or influence the active site of the enzyme (15).

The variation observed in the clinical phenotype may be attributed to the location of the mutations on the protein domains. Thus, Navon Elkan et al (1) found that glycine at residue 47 of ADA2 was highly conserved in a variety of sequenced species, thus suggesting that its substitution with arginine is predicted to be highly damaging. In particular, Gly47Arg and Gly47Val possibly affect the stability of homodimers or their individual subunits and seem to be the most prominent mutations causing PAN. However, the disease severity was found to be highly varied from mild type of the disease limited to the skin, without any constitutional symptoms to severe, which was finally fatal in some cases (1). As regards the study conducted by Gibson et al (18), the authors suggested that Arg8Trp may have benign consequences and both Leu351Gln and Ala357Thr probably damaging ones. It has been suggested that the clinical significance of novel variants cannot be assessed from their allele frequencies only (21). Therefore, their consequences on protein function must be analyzed by proper biochemical assays and/or in silico analysis using bioinformatics (22).

Özen et al (23) attempted to perform a possible genotype-phenotype correlation and reported that dimerization domain mutations are associated with PAN-like phenotype, while catalytic domain mutations are associated with hematological manifestations. Furthermore, homozygous G47R mutation has been detected in a Jewish patient diagnosed with HHV-8-negative Castelman disease (24). In another clinical study involving 10 DADA2 patients from The Netherlands and Belgium, homozygosity for R169Q mutation was associated with the presence of cytopenia (25). In two patients carrying a homozygous deletion of the locus 22q11.1, harboring both copies of the IL-17 receptor A (IL17RA) and the $C E C R l$ gene, muco-cutaneous infections and dermatitis were observed (26). By contrast, Gibson et al (18) were not able to correlate genotype to phenotype in nine children diagnosed with DADA2, thus assuming that apart from the ADA2 mutations other factors such as modifying genes, epigenetic modifications and possibly environmental factors are also involved in the pathogenesis of DADA2 and disease expressivity. Therefore, further studies are warranted in order to better understand the genotype-phenotype correlation in patients with DADA2.

The findings presented by Gibson et al (18) are supportive of previous reports that the disease under investigation appears an extensive genotypic and phenotypic 
variability, which cannot be explained by the importance of each causal mutation regarding the protein function $(1,6)$. The published case series of patients with DADA2 revealed a large phenotypic variability that cannot be fully explained by the impact of causal mutations on protein function. To the best of our knowledge, this is the first study to evaluate the structural significance of the ADA2 novel mutations under discussion. It was concluded that Arg8Trp and Gly47Arg mutations are affecting the position and interaction of the dimer associated NH1 helical structure and therefore dimer formation and stabilization, while Leu351Gln and Ala357Thr influence the metal coordination in the active site. This information may aid in the further interpretation of the recent findings of Gibson et al (18) from the structural biology point of view and justifies that screening ADA2 in children with various types of pediatric vasculitis may enable an earlier diagnosis of DADA2. Moreover, it is important to be aware of the identity of these monogenic diseases with vasculitic features, taking into account that any diagnostic delay may be considerable and the alternative treatments for these diseases differs than classical vasculitides.

\section{Acknowledgements}

Not applicable.

\section{Funding}

No funding was received.

\section{Availability of data and materials}

The datasets used and/or analyzed during the current study are available from the corresponding author on reasonable request.

\section{Authors' contributions}

EE, CM, MM and GNG conceived and designed the study and drafted the manuscript. EE, GNG, CM, DAS and MIZ searched the literature. EE, MM, DAS and MIZ analyzed and interpreted the data. MIZ and DAS critically revised the manuscript. All authors have read and approved the final manuscript.

\section{Ethics approval and consent to participate}

Not applicable.

\section{Patient consent for publication}

Not applicable.

\section{Competing interests}

DAS is the Editor-in-Chief for the journal, but had no personal involvement in the reviewing process, or any influence in terms of adjudicating on the final decision, for this article. All the other authors declare that they have no competing interests.

\section{References}

1. Navon Elkan P, Pierce SB, Segel R, Walsh T, Barash J, Padeh S, Zlotogorski A, Berkun Y, Press JJ, Mukamel M, et al: Mutant adenosine deaminase 2 in a polyarteritis nodosa vasculopathy. $\mathrm{N}$ Engl J Med 370: 921-931, 2014.

2. Zhou Q, Yang D, Ombrello AK, Zavialov AV, Toro C, Zavialov AV Stone DL, Chae JJ, Rosenzweig SD, Bishop K, et al: Early-onset stroke and vasculopathy associated with mutations in ADA2. N Engl J Med 370: 911-920, 2014.

3. Caorsi R, Penco F, Schena F and Gattorno M: Monogenic polyarteritis: The lesson of ADA2 deficiency. Pediatr Rheumatol Online J 14: 51, 2016.

4. Francès C, Papo T, Wechsler B, Laporte JL, Biousse V and Piette JC: Sneddon syndrome with or without antiphospholipid antibodies. A comparative study in 46 patients. Medicine (Baltimore) 78: 209-219, 1999.

5. Van Eyck L Jr, Hershfield MS, Pombal D, Kelly SJ, Ganson NJ, Moens L, Frans G, Schaballie H, De Hertogh G, Dooley J, et al: Hematopoietic stem cell transplantation rescues the immunologic phenotype and prevents vasculopathy in patients with adenosine deaminase 2 deficiency. J Allergy Clin Immunol 135: 283-287.e5, 2015.

6. Ben-Ami T, Revel-Vilk S, Brooks R, Shaag A, Hershfield MS, Kelly SJ, Ganson NJ, Kfir-Erenfeld S, Weintraub M, Elpeleg O, et al: Extending the Clinical Phenotype of Adenosine Deaminase 2 Deficiency. J Pediatr 177: 316-320, 2016.

7. Cipe FE, Aydogmus C, Serwas NK, Keskindemirci G and Boztug K: Novel mutation in CECR1 leads to deficiency of ADA2 with associated neutropenia. J Clin Immunol 38: 273-277, 2018.

8. Westendorp WF, Nederkoorn PJ, Aksentijevich I, Hak AE, Lichtenbelt KD and Braun KP: Unexplained early-onset lacunar stroke and inflammatory skin lesions: Consider ADA2 deficiency. Neurology 84: 2092-2093, 2015.

9. Elbracht M, Mull M, Wagner N, Kuhl C, Abicht A, Kurth I, Tenbrock K and Häusler M: Stroke as initial manifestation of adenosinedeaminase 2 deficiency. Neuropediatrics 48: 111-114, 2017.

10. Sahin S, Adrovic A, Barut K, Ugurlu S, Turanli ET, Ozdogan H and Kasapcopur O: Clinical, imaging and genotypical features of three deceased and five surviving cases with ADA2 deficiency. Rheumatol Int 38: 129-136, 2018.

11. Bulut E, Erden A, Karadag O, Oguz KK and Ozen S: Deficiency of adenosine deaminase 2; special focus on central nervous system imaging. J Neuroradiol 46: 193-198, 2019.

12. Zavialov AV, Gracia E, Glaichenhaus N, Franco R, Zavialov AV and Lauvau G: Human adenosine deaminase 2 induces differentiation of monocytes into macrophages and stimulates proliferation of $\mathrm{T}$ helper cells and macrophages. J Leukoc Biol 88: 279-290, 2010.

13. Zavialov AV and Engström A: Human ADA2 belongs to a new family of growth factors with adenosine deaminase activity. Biochem J 391: 51-57, 2005 .

14. Haskó G, Linden J, Cronstein B and Pacher P: Adenosine receptors: Therapeutic aspects for inflammatory and immune diseases. Nat Rev Drug Discov 7: 759-770, 2008.

15. Meyts I and Aksentijevich I: Deficiency of Adenosine Deaminase 2 (DADA2): Updates on the Phenotype, Genetics, Pathogenesis, and Treatment. J Clin Immunol 38: 569-578, 2018.

16. Wilson DK, Rudolph FB and Quiocho FA: Atomic structure of adenosine deaminase complexed with a transition-state analog: Understanding catalysis and immunodeficiency mutations. Science 252: 1278-1284, 1991.

17. Charlab R, Valenzuela JG, Andersen J and Ribeiro JM: The invertebrate growth factor/CECR1 subfamily of adenosine deaminase proteins. Gene 267: 13-22, 2001.

18. Gibson KM, Morishita KA, Dancey P, Moorehead P, Drögemöller B, Han X, Graham J, Hancock REW, Foell D, Benseler S, et al; PedVas Investigators Network: Identification of novel adenosine deaminase 2 gene variants and varied clinical phenotype in pediatric vasculitis. Arthritis Rheumatol 71: 1747-1755, 2019.

19. Schrödinger LLC: The PyMOL Molecular Graphics System 2016 version 2.2. pymol.org/2/support.html. Accessed March 5, 2019.

20. Kinoshita T, Nakanishi I, Terasaka T, Kuno M, Seki N, Warizaya M, Matsumura H, Inoue T, Takano K, Adachi H, et al: Structural basis of compound recognition by adenosine deaminase. Biochemistry 44: 10562-10569, 2005. 
21. Meyts I, Bosch B, Bolze A, Boisson B, Itan Y, Belkadi A Pedergnana V, Moens L, Picard C, Cobat A, et al: Exome and genome sequencing for inborn errors of immunity. J Allergy Clin Immunol 138: 957-969, 2016.

22. Caorsi R, Penco F, Grossi A, Insalaco A, Omenetti A, Alessio M, Conti G, Marchetti F, Picco P, Tommasini A, et al: ADA2 deficiency (DADA2) as an unrecognised cause of early onset polyarteritis nodosa and stroke: A multicentre national study. Ann Rheum Dis 76: 1648-1656, 2017.

23. Özen S, Batu ED, Taskiran EZ, Özkara HA, Ünal S, Güleray N, Erden A, Karadağ Ö, Gümrük F, et al: A monogenic disease with a variety of phenotypes: deficiency of adenosine deaminase 2 . J Rheum: May 1, 2019 (Epub ahead of print).

24. Van Eyck L, Liston A and Wouters C: Mutant ADA2 in vasculopathies. N Engl J Med 371: 480, 2014.
25. Van Montfrans JM, Hartman EA, Braun KP, Hennekam EA, Hak EA, Nederkoorn PJ, Westendorp WF, Bredius RG, Kollen WJ, Schölvinck EH, et al: Phenotypic variability in patients with ADA2 deficiency due to identical homozygous R169Q mutations. Rheumatology (Oxford) 55: 902-910, 2016.

26. Fellmann F, Angelini F, Wassenberg J, Perreau M, Arenas Ramirez N, Simon G, Boyman O, Demaria O, Christen-Zaech S, Hohl D, et al: IL-17 receptor A and adenosine deaminase 2 deficiency in siblings with recurrent infections and chronic inflammation. J Allergy Clin Immunol 137: 1189-1196.e2, 2016.

This work is licensed under a Creative Commons

Attribution-NonCommercial-NoDerivatives 4.0 International (CC BY-NC-ND 4.0) License. 